\title{
Boundary-Improved Distance Vector-Hop Localization Method with Multipower Correction for Wireless Sensor Networks
}

\author{
Chwan-Lu Tseng, ${ }^{*}$ Foun-Yuan Liu, Cheng-Han Lin, and Ching-Yin Lee ${ }^{1}$ \\ Department of Electrical Engineering, National Taipei University of Technology \\ 1, Sec. 3, Zhongxiao E. Rd., Taipei 10608, Taiwan, R.O.C. \\ ${ }^{1}$ Department of Electrical Engineering, Tungnan University, \\ 152, Sec. 3, Beishen Rd., Shenkeng Dist., New Taipei City 22202, Taiwan, R.O.C.
}

(Received October 17, 2016; accepted December 5, 2016)

Keywords: wireless sensor networks, localization, DOI, multidimensional scaling, multipower transmission

As wireless sensor networks (WSNs) become more advanced, they are gradually applied to various fields, such as medical monitoring, and receive increased attention because of their great potential. WSN localization methods are crucial for numerous applications. To increase localization accuracy, a method is proposed that calculates the number of hops between nodes using the degree of irregularity model. The amorphous method is then adopted to calculate the average distance, and multidimensional scaling is used to estimate the coordinates of unknown nodes. Finally, boundary correction and multipower transmission techniques are adopted to reduce the error of estimation. This integrated localization method reduces localization errors which may occur at each step of distance vector (DV)-hop localization. The proposed method-boundaryimproved amorphous localization with multipower multidimensional scaling (BIA-MMS) substantially improved the localization accuracy compared with other localization methods when tested through simulations.

\section{Introduction}

Wireless sensor networks (WSNs) were developed two decades ago by the US military to detect objects on the battlefield. The objective of the "smart dust" research project at the University of California at Berkeley, in collaboration with the US Department of Defense, was to develop miniature sensors and a network that could connect them. ${ }^{(1,2)}$ This dust could be used to reduce the cost of continuous monitoring of hostile environments and prevent accidents. Microelectromechanical systems manufacturing and embedded processor technology have recently been used to develop energy-efficient single-chip microcomputers. Consequently, numerous research institutions and industrial companies have become interested in developing applications that use WSNs, including the monitoring of oriental fruit flies, ${ }^{(3)}$ air pollution, ${ }^{(4)}$ motor faults, ${ }^{(5)}$ volcanic eruptions, ${ }^{(6)}$ and home healthcare. ${ }^{(7)}$ For some applications, localization accuracy is crucial, and has thus been the focus of much research. Some of the WSNs that can estimate locations most

*Corresponding author: e-mail: cltseng@ee.ntut.edu.tw http://dx.doi.org/10.18494/SAM.2017.1489 
precisely use high-cost nodes, which locate each other using a global positioning system (GPS), but these negate the low-cost advantage of WSNs. Consequently, range-based and range-free localization schemes that do not use expensive positioning devices are preferable when cost is a concern. A range-based localization scheme assumes that all nodes are equipped with directional antennas to find directions and estimate distances. A range-free localization scheme does not use expensive directional antennas but only omnidirectional ones. Locations are often estimated by range-free devices using the received signal strength indicator (RSSI). Theoretically, the RSSI indicates the distance between the transmitting and receiving nodes. In practice, however, the environment can easily affect the RSSI and the localization results have large errors. To decrease this error, hop counts are used instead. The distance vector (DV)-hop algorithm ${ }^{(8)}$ is a popular hop count localization algorithm. On the basis of the number of hops between a node and an anchor node, location coordinates can be estimated. However, this approach requires high-density deployment to locate node coordinates precisely. Recently, researchers have proposed variations of the DV-hop technique. The modified DV-hop method $^{(9)}$ entails a weighted hop-distance estimation approach. In the amorphous method, ${ }^{(10,11)}$ the hop count modification was introduced when the distances between unknown nodes and anchor nodes were calculated. Reference 12 introduced a twice-weighted DV-hop localization algorithm to more accurately estimate hop distances in which the RSSI was not used. To consider path loss, Ref. 13 improved localization accuracy by using the RSSI ratio model to modify hop distance estimation.

Although these methods substantially mitigated localization errors, the large errors that often occur in the boundary regions of uniformly, randomly deployed networks have rarely been addressed. Similar to the aforementioned methods, the method proposed in this paper uses the hop count from node to node to estimate distances and then determine coordinates. This method, called boundary-improved amorphous localization with multipower multidimensional scaling (BIAMMS), involves applying the degree of irregularity (DOI) model, the shortest path algorithm, multidimensional scaling (MDS), multipower method, and the boundary improvement algorithm to estimate distances and reduce localization errors. Part of preliminary work has been published. ${ }^{(14)}$ Compared with other localization methods, simulation results demonstrated that BIA-MMS estimated coordinates more accurately than other methods did.

\section{Preliminaries}

\subsection{DV-hop localization method}

The DV-hop localization algorithm ${ }^{(8)}$ is classified as a range-free localization scheme. If the anchor node cannot send a packet to the target node directly, the redirect hop count is calculated and used to estimate the average distance based on the average hop distance. When distances between the target node and more than three anchor nodes are obtained, the coordinates of the target node can be estimated. Assuming that $d_{i}$ is the estimated distance of the target node with the coordinates $(x, y)$ from the anchor node $i$ with the coordinates $\left(x_{i}, y_{i}\right), i=1, \ldots, m$,

$$
\begin{gathered}
\left(x_{1}-x\right)^{2}+\left(y_{1}-y\right)^{2}=d_{1}^{2}, \\
\vdots \\
\left(x_{m}-x\right)^{2}+\left(y_{m}-y\right)^{2}=d_{m}^{2} .
\end{gathered}
$$


Subtracting the final equation in Eq. (1) from the rest of the equations, we have

$$
\begin{gathered}
2 x\left(x_{1}-x_{m}\right)+2 y\left(y_{1}-y_{m}\right)=d_{m}^{2}-d_{1}^{2}+x_{1}^{2}-x_{m}^{2}+y_{1}^{2}-y_{m}^{2}, \\
\vdots \\
2 x\left(x_{m-1}-x_{m}\right)+2 y\left(y_{m-1}-y_{m}\right)=d_{m}^{2}-d_{m-1}^{2}+x_{m-1}^{2}-x_{m}^{2}+y_{m-1}^{2}-y_{m}^{2} .
\end{gathered}
$$

Rewriting Eq. (2) in matrix-vector form gives

$$
A x=b,
$$

where $\boldsymbol{x}=\left[\begin{array}{ll}x y\end{array}\right]^{\mathrm{T}}$, and $\boldsymbol{A}=\left[\begin{array}{cc}x_{1}-x_{m} & y_{1}-y_{m} \\ \vdots & \vdots \\ x_{m-1}-x_{m} & y_{m-1}-y_{m}\end{array}\right], \boldsymbol{b}=\left[\begin{array}{c}\left(d_{m}^{2}-d_{1}^{2}+x_{1}^{2}-x_{m}^{2}+y_{1}^{2}-y_{m}^{2}\right) / 2 \\ \vdots \\ \left(d_{m}^{2}-d_{m-1}^{2}+x_{m-1}^{2}-x_{m}^{2}+y_{m-1}^{2}-y_{m}^{2}\right) / 2\end{array}\right]$.

By the projection method, the least-squares solution can be found:

$$
\hat{\boldsymbol{x}}=\left(\boldsymbol{A}^{\mathrm{T}} \boldsymbol{A}\right)^{-1} \boldsymbol{A}^{\mathrm{T}} \boldsymbol{b} .
$$

\subsection{Modified DV-hop localization method}

The modified DV-hop localization $\operatorname{method}^{(9)}$ entails a new calculation of average hop distance (hop size), which is composed of two parts: global hop distance and local hop distance. The local distance $C_{l}^{i, j}$ between the anchor nodes $i$ and $j$ is defined as

$$
C_{l}^{i, j}=\sqrt{\left(x_{i}-x_{j}\right)^{2}+\left(y_{i}-y_{j}\right)^{2}} / h_{i j}
$$

where $h_{i j}$ is the hop count from the anchor node $i$ to the anchor node $j$. The global distance $C_{g}^{i}$ of the anchor node $i$ is defined as

$$
C_{g}^{i}=\sum_{i \neq j} \sqrt{\left(x_{i}-x_{j}\right)^{2}+\left(y_{i}-y_{i}\right)^{2}} / \sum_{i \neq j} h_{i j}
$$

The effective average hop distance is then calculated as a weighted average using

$$
C_{e f f}^{i, j}=\alpha C_{g}^{i}+(1-\alpha) C_{l}^{i, j}
$$

where $0<\alpha<1$ is a positive weighted coefficient. Using Eq. (7), the distance from the anchor node to the target node can be simply obtained from the product of the hop distance and hop count.

\subsection{DOI model}

Because of environmental interference, the range of a radio signal is usually not circular. This type of irregularity is a common phenomenon in WSNs. To model this irregularity, Ref. 15 
proposed a DOI model. Assuming that $\mathrm{d}$ is the distance between the transmitting and receiving nodes, and that $d_{0}$ is the reference distance, the receiving power $P_{r}(d)$ can be defined as

$$
P_{r}(d)=P_{t}-P_{L}\left(d_{0}\right)-10 \eta \log _{10}\left(\frac{d}{d_{0}}\right) \times D_{i},
$$

where $P_{t}$ is the transmitting power and $\eta$ is the exponent of path loss that depends on the propagation environment. The typical range of $\eta$ is $2 \leq \eta \leq 4$ for outdoor environments and $4 \leq \eta \leq$ 6 for indoor environments. Table 1 lists some examples of $\eta$ in different environments.

In Eq. (8), $D_{i}$ is the coefficient that represents the grade of path loss in the direction of degree $i$, which is defined as

$$
D_{i}= \begin{cases}1 & \text { if } i=0 \\ D_{i-1} \pm \text { Rand } \times D O I & \text { if } 0<i<360, i \text { is a positive integer }\end{cases}
$$

provided $\left|D_{0}-D_{359}\right| \leq D O I$, where $0 \leq D O I \leq 1$ is a pre-specified number to indicate the degree of irregularity. Using Eqs. (8) and (9), the transmission radius in the direction $i$ can be calculated as

$$
\phi(i)=d_{0} \times 10^{\left(P_{t}-P_{r}(d)-P_{L}\left(d_{0}\right) / 10 \times \eta \times D_{i}\right)} .
$$

Different DOI values in Eq. (10) reflect the different possible transmission characteristics. Figure 1 illustrates the transmission irregularity when DOI is $0.00,0.05$, and 0.07 . When DOI is 0 , we obtain a perfectly circular transmission. When the DOI is increased, the degree of transmission irregularity increases.

Table 1

Typical values of $\eta$.

\begin{tabular}{lc}
\hline Environment & Exponent of path loss $\eta$ \\
\hline Free space & 2 \\
Urban area & $2.7-3.5$ \\
Suburban area & $3-5$ \\
Indoor (line-of-sight) & $1.6-1.8$ \\
Indoor (not line-of-sight) & $4-6$ \\
\hline
\end{tabular}

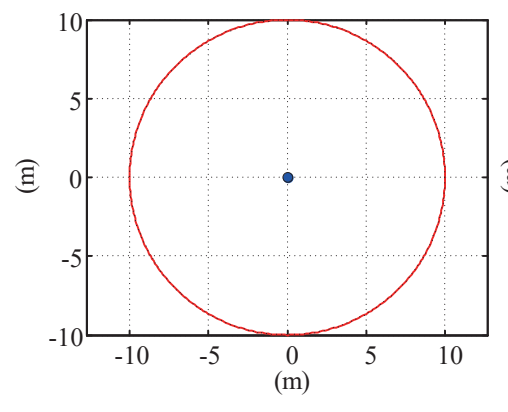

$\mathrm{DOI}=0.00$

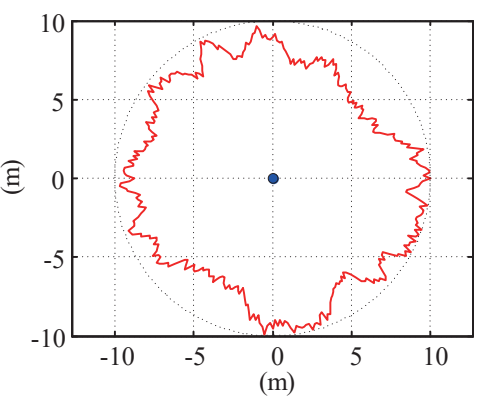

$\mathrm{DOI}=0.05$

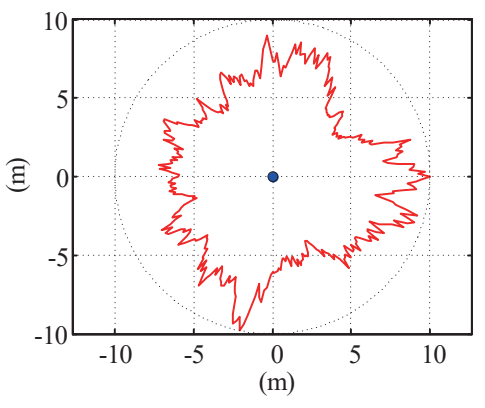

$\mathrm{DOI}=0.07$

Fig. 1. (Color online) Irregular transmission radii found using the DOI model. 


\section{BIA-MMS Method}

The purpose of the localization method proposed in this paper is to accurately estimate positions in WSNs. The localization procedure follows the steps of DV-hop localization: (1) calculate the hop count, (2) estimate the hop distance, and (3) find the node coordinates. It differs from the traditional DV-hop method in the sense that it adopts a statistical method to find the average hop distance, ${ }^{(16,17)}$ the amorphous method ${ }^{(10,11)}$ to calculate average hop count, and the MDS method ${ }^{(18,19)}$ to estimate coordinates. Additionally, boundary improvement and multipower transmission techniques are introduced to further improve the localization accuracy. By using fixed anchor nodes, the method estimates the coordinates of ordinary sensor nodes. To sum up, the integration localization method proposed by this work can reduce the localization errors which may occur at each step of the DV-hop localization. Moreover, the boundary correction scheme and the multipower method are adopted to further reduce the overall localization error.

Initially, the concept of modeling radio irregularity detailed in Ref. 15 is used to establish the DOI model and simulate the wireless transmissions of WSNs. In addition, WSNs are deployed randomly to meet the $100 \%$ coverage requirement. After this deployment, the sink sends command packets to all anchor nodes and the nodes to be located. When it receives the packet, each node collects the ID numbers of its neighbor nodes and returns them to the sink, which establishes the $n$ $\times n$ adjacency matrix $\boldsymbol{C}$. The element $\boldsymbol{C}(i, j)$ is defined as

$$
C(i, j)= \begin{cases}1, & \text { if } j \text { is a neighbor node of } i \\ 0, & i=j \\ \infty, & \text { otherwise }\end{cases}
$$

where $i$ is the ID of the starting node, $j$ is the ID of the destination node $(i, j=1, \ldots, n)$, and $n$ is the number of nodes in the network. $\boldsymbol{C}(i, j)$ indicates the connectivity between nodes $i$ and $j$. If node $j$ lies within the communication range of node $i, \boldsymbol{C}(i, j)=1$; if they cannot connect, $\boldsymbol{C}(i, j)=\infty$.

Once $\boldsymbol{C}$ is found, the number of hops can be calculated from the shortest path matrix $\boldsymbol{W}$ (or hop count matrix) between all nodes by using the Floyd-Warshall algorithm. ${ }^{(20)}$ This algorithm uses $\boldsymbol{C}$ as an initial structure matrix. The hop count matrix at step $k$ is denoted by $\boldsymbol{W}_{k}$, and $\boldsymbol{W}_{0}=C$. When the algorithm proceeds to step $k, k$ is the relay node. In this case, the hop count information at step $k$ is compared with that at step $k-1$. If the hop count at $k$ is larger, let $\boldsymbol{W}_{k}(i, j)=\boldsymbol{W}_{k-1}(i, j)$; if the hop count at $k$ is smaller, let $\boldsymbol{W}_{k}(i, j)=\boldsymbol{W}_{k-1}(i, k)+\boldsymbol{W}_{k-1}(k, j)$. Therefore, the updated equation of the Floyd-Warshall algorithm is

$$
\boldsymbol{W}_{k}(i, j)=\min \left\{\boldsymbol{W}_{k-1}(i, j), \boldsymbol{W}_{k-1}(i, k)+\boldsymbol{W}_{k-1}(k, j)\right\}
$$

The minimum hop count matrix, denoted by $\boldsymbol{W}(i, j)$ using the Floyd-Warshall algorithm, can be obtained by dynamic programming.

To estimate the distance between nodes, the hop count and transmission distance are required. Also, the average distance of each hop is essential to calculate the distance accurately. It is assumed that the average network connectivity is known a priori. Because this work adopts random WSN deployment and assumes that the number of nodes within a transmission radius has a Poisson distribution, ${ }^{(16)}$ the Poisson distribution method is used to estimate the average number of neighboring nodes for each sensor node. Using this network node density, the method proposed by 
Kleinrock and Silvester ${ }^{(17)}$ is used to calculate the average distance of each sensing node hop $d_{\text {hop }}$ :

$$
d_{\text {hop }}=R\left[1+e^{-N}-\int_{-1}^{1} e^{-\frac{N}{\pi}\left(\cos ^{-1}(t)-t \sqrt{1-t^{2}}\right)} d t\right],
$$

where $N$ is the expected number of nodes within the transmission radius $R$.

Once the expected distance of one hop is obtained, it is possible to update the estimated distance between nodes. Traditionally, the estimated distance between nodes $i$ and $j$ is calculated using $\boldsymbol{W}(i, j) \times R$, where $\boldsymbol{W}(i, j)$ is the hop count between the starting node $i$ and the destination node $j$. From this, it can be concluded that the localization error is due to the multiplication of the hop count error by $R$, which is generally large. Equation (13) proposed a reasonable estimation of the average distance which is usually smaller than $R$ and results in a smaller distance error from node $i$ to node $j$. To calculate the average number of hops within the network and thus improve the accuracy of the estimated location, the amorphous method ${ }^{(12)}$ is used. In Ref. 11, a 0.5 shift was introduced to each element in $\boldsymbol{W}$. This updates $\boldsymbol{W}$ to

$$
\hat{\boldsymbol{W}}(i, j)=\frac{\sum_{k \in n b r(i)} \boldsymbol{W}(k, j)+\boldsymbol{W}(i, j)}{|n b r(i)|+1}-0.5, \quad j=1,2, \ldots, n,
$$

where $n b r(i)$ is the set of neighbor nodes of the node $i,|n b r(i)|$ is the number of nodes in $n b r(i)$, and $\hat{\boldsymbol{W}}(i, j)$ is the element $(i, j)$ of the updated hop count matrix $\hat{\boldsymbol{W}}$. Consequently, the estimated distance can then be updated to $\hat{\boldsymbol{W}}(i, j) \times d_{\text {hop }}$ and the distance matrix $\boldsymbol{L}$ can be found using

$$
\boldsymbol{L}=\hat{\boldsymbol{W}} \times d_{\text {hop }} .
$$

Instead of the traditional least-squares method, this study proposes the use of the MDS method $^{(18)}$ to calculate the coordinates of a node when information about neighboring nodes within the communications range is known. Also, the double-centering method introduces an inner product matrix $\boldsymbol{B}$ :

$$
\boldsymbol{B}=\boldsymbol{X} \boldsymbol{X}^{\mathrm{T}}=\frac{1}{2} \boldsymbol{J} \boldsymbol{L}^{2} \boldsymbol{J}
$$

where $\boldsymbol{J}=\boldsymbol{I}-\left([1 \cdots 1]_{1 \times n}^{\mathrm{T}} \times[1 \cdots 1]_{1 \times n}\right) / n$. The new centered matrix $\boldsymbol{X}^{*}$ can be calculated by subtracting the column means of $\boldsymbol{X}$ :

$$
\boldsymbol{X}^{*}=\boldsymbol{X}-\boldsymbol{H} \boldsymbol{C}=\boldsymbol{X}-\left(\boldsymbol{H} \boldsymbol{H}^{\mathrm{T}} \boldsymbol{X}\right) / n,
$$

where $\boldsymbol{H}=\left[\begin{array}{lll}1 & \cdots & 1\end{array}\right]^{\mathrm{T}}$ and $\boldsymbol{C}=\left(\boldsymbol{H}^{\mathrm{T}} \boldsymbol{X}\right) / n$. Thus, the new inner product matrix $\boldsymbol{B}^{*}$ of the centered configuration can be defined as

$$
\begin{aligned}
\boldsymbol{B}^{*} & =\boldsymbol{X}^{*} \boldsymbol{X}^{* \mathrm{~T}} \\
& =(\boldsymbol{X}-\boldsymbol{H C})(\boldsymbol{X}-\boldsymbol{H C})^{\mathrm{T}} \\
& =\left(\boldsymbol{X}-\boldsymbol{H} \boldsymbol{H}^{\mathrm{T}} \boldsymbol{X} / n\right)\left(\boldsymbol{X}^{\mathrm{T}}-\boldsymbol{X}^{\mathrm{T}} \boldsymbol{H} \boldsymbol{H}^{\mathrm{T}} / n\right) \\
& =\boldsymbol{B}-\boldsymbol{B} \boldsymbol{H} \boldsymbol{H}^{\mathrm{T}} / n-\boldsymbol{H} \boldsymbol{H}^{\mathrm{T}} \boldsymbol{B} / n+\boldsymbol{H} \boldsymbol{H}^{\mathrm{T}} \boldsymbol{B} \boldsymbol{H} \boldsymbol{H}^{\mathrm{T}} / n^{2} .
\end{aligned}
$$


Using direct matrix manipulation, the elements of $\boldsymbol{B}^{*}$ can be written as

$$
b_{i j}^{*}=b_{i j}-\frac{1}{n} \sum_{k=1}^{n} b_{i k}-\frac{1}{n} \sum_{k=1}^{n} b_{k j}+\frac{1}{n^{2}} \sum_{k=1}^{n} \sum_{l=1}^{n} b_{k l} .
$$

Note that $b_{j k}=\left(d_{i j}^{2}+d_{i k}^{2}-d_{j k}^{2}\right) / 2$, and that $d_{i j}, d_{i k}$, and $d_{j k}$ are restricted by the law of cosines: $\cos \theta_{j i k}=\left(d_{i j}^{2}+d_{i k}^{2}-d_{j k}^{2}\right) / 2 d_{i j} d_{i k}$. Taking the singular value decomposition of $\boldsymbol{B}^{*}$, we have

$$
\boldsymbol{B}^{*}=\boldsymbol{U} \Sigma \boldsymbol{V}^{\mathrm{T}}
$$

where $\boldsymbol{U}$ and $\boldsymbol{V}$ are normal matrices, and $\boldsymbol{\Sigma}$ is in the form

$$
\boldsymbol{\Sigma}=\left[\begin{array}{cc}
\boldsymbol{\Lambda}_{r} & 0 \\
0 & 0
\end{array}\right]_{n \times n}, \boldsymbol{\Lambda}_{r}=\left[\begin{array}{ccc}
\sigma_{1} & 0 & 0 \\
0 & \ddots & 0 \\
0 & 0 & \sigma_{r}
\end{array}\right]_{r \times r}
$$

Without loss of generality, we assume that $\sigma_{i}$ satisfies $\sigma_{1} \geq \sigma_{2} \geq \ldots \geq \sigma_{r}>0$. Choosing two singular values $\sigma_{1}$ and $\sigma_{2}$ and their associated singular vectors, we can calculate the dissimilarity matrix:

$$
\boldsymbol{E}=\boldsymbol{U} \times\left[\begin{array}{cc}
\sigma_{1} & 0 \\
0 & \sigma_{2} \\
\vdots & \vdots \\
0 & 0
\end{array}\right]_{n \times 2}
$$

The estimated coordinate of node $i$ corresponds to row $i$ of matrix $\boldsymbol{E}$. The element $\boldsymbol{E}(i, 1)$ is the estimated $x$-axis coordinate $x_{e s t}^{i}$, and the element $\boldsymbol{E}(i, 2)$ is the estimated $y$-axis coordinate $y_{e s t}^{i}$. The method proposed in Ref. 19 to improve localization accuracy by solving the nonlinear equations of rotation and translation was adopted.

After the estimated coordinates were found, they were plotted to illustrate the error in the estimated position (Fig. 2). In Fig. 2, dark dots indicate the actual positions of nodes; grey dots indicate the estimated positions; white dots indicate the estimated positions near the boundary; stars denote the anchor nodes. The figure demonstrates that the localization error appears to be largest near the boundary of the deployment area. Hence, the following rule is proposed to reduce the error:

$$
\hat{\boldsymbol{E}}(i, j)= \begin{cases}N S, & \text { if } \boldsymbol{E}(i, j)>N S, \forall i \in\{1, \ldots, n\}, \forall j \in\{1,2\} \\ 0, & \text { if } \boldsymbol{E}(i, j)<0, \forall i \in\{1, \ldots, n\}, \forall j \in\{1,2\} \\ \boldsymbol{E}(i, j), & \text { otherwise }\end{cases}
$$

where NS is the network size (deployment area). Note that the reference origin in Fig. 2 is in the lower lefthand corner. If the estimated coordinate is outside the deployment area, it is replaced by $N S$ or zero.

In general, hop-based localization methods have similar drawbacks. The estimated distance between the node and the anchor node is based on hop count. If the sensor node is far from the 


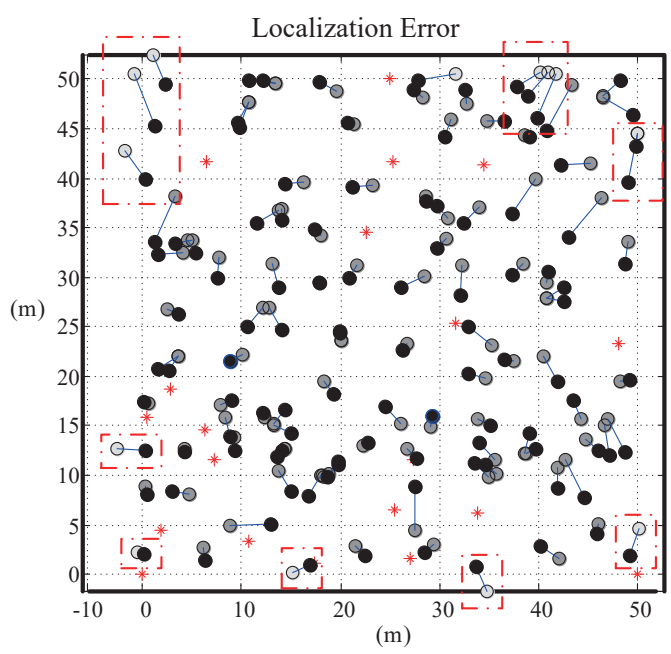

Fig. 2. (Color online) Indication of the large boundary localization error.

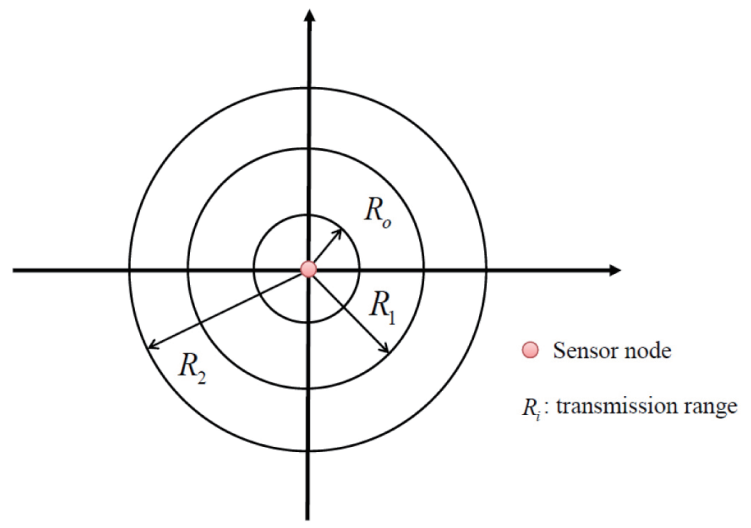

Fig. 3. (Color online) Multipower transmission.

anchor node, the hop count is high and this causes a greater accumulation of error in the distance calculation. Therefore, the nodes that are farther from the anchor node must be located differently. Accordingly, multipower localization ${ }^{(21)}$ is employed, within which three transmission powers (with correspondingly different transmission ranges) are used to send packets (Fig. 3).

Three hop count matrices $\boldsymbol{W}_{R_{0}}, \boldsymbol{W}_{R_{1}}$, and $\boldsymbol{W}_{R_{2}}$, and thus three coordinate matrices $\hat{\boldsymbol{E}}_{R_{0}}, \hat{\boldsymbol{E}}_{R_{1}}$, and $\hat{\boldsymbol{E}}_{R_{2}}$, are then obtained. Nodes that are more than three hops from the anchor node are collected from $\boldsymbol{W}$, and the node sequence is rearranged by the hop count in descending order. By selecting half of the sequence with larger hop counts, the coordinates are then modified using the following:

$$
\hat{\boldsymbol{E}}_{m p}(i, j)=\frac{1}{3}\left(\hat{\boldsymbol{E}}_{R_{0}}(i, j)+\hat{\boldsymbol{E}}_{R_{1}}(i, j)+\hat{\boldsymbol{E}}_{R_{2}}(i, j)\right), \quad j=1,2 .
$$

For simplicity, in this paper, we denote the proposed boundary-improved amorphous localization algorithm with multipower MDS by BIA-MMS. This method is compared in this paper with two methods in which multipower modification is not used: the amorphous MDS (AMDS) method (in which boundary improvements are not made), and the boundary-improved MDS (BIA-MDS) method (which has no multipower modification). The normalized error in the estimated location is defined as

$$
M_{R}^{i}=\frac{\sqrt{\left(x_{\text {est }}^{i}-x_{\text {real }}^{i}\right)^{2}+\left(y_{\text {est }}^{i}-y_{\text {real }}^{i}\right)^{2}}}{R},
$$

and the average error of estimation as

$$
M\left(M_{R}\right)=\frac{\sum_{i=1}^{n} M_{R}^{i}}{n} .
$$


Comparing AMDS and BIA-MDS revealed that adding boundary improvement resulted in a smaller average error of estimation [Fig. 4(a)]. Notably, the unit (round) of the $x$-axis in Fig. 4 is the number of simulations and the unit $(\mathrm{R})$ of the $y$-axis is the transmission range. Moreover, Eqs. (13) and (15) indicate that the transmission radius plays an important role in the calculation of localization error, so this paper uses a multipower method to refine the estimated coordinates. Compared with AMDS and BIA-MDS, the BIA-MMS method yielded a lower average error of estimation [Fig. 4(b)]. In summary, Fig. 5 shows the flowchart of the proposed BIA-MMS localization method.

\section{Numerical Simulations}

To demonstrate the effectiveness of the proposed method, other localization methods, namely, DV-hop, ${ }^{(8)}$ Im DV-hop, ${ }^{(9)}$ amorphous, ${ }^{(10)}$ grid-scan, ${ }^{(22)}$ and AMDS, were used for comparison. Matlab simulation software was used and simulation parameter settings were as follows:

(1) Deployment area $=50 \times 50 \mathrm{~m}^{2}$, random deployment;

(2) Number of nodes $=130$;

(3) Transmission range $(\mathrm{R})=10 \mathrm{~m}$;

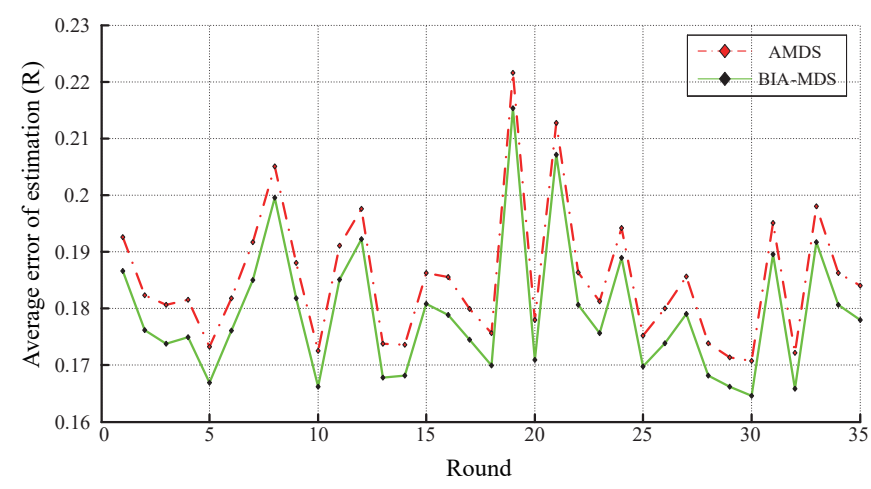

(a)

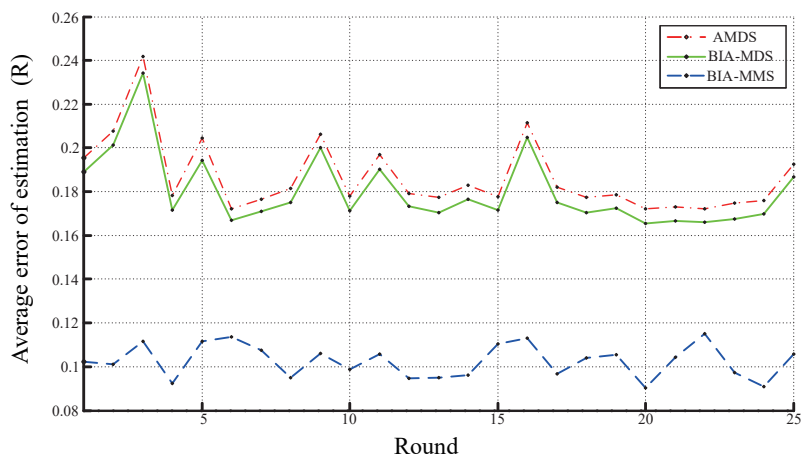

(b)

Fig. 4. (Color online) (a) Comparison of average error of estimation between AMDS and BIA-MDS. (b) Comparison of average error of estimation among AMDS, BIA-MDS, and BIA-MMS.

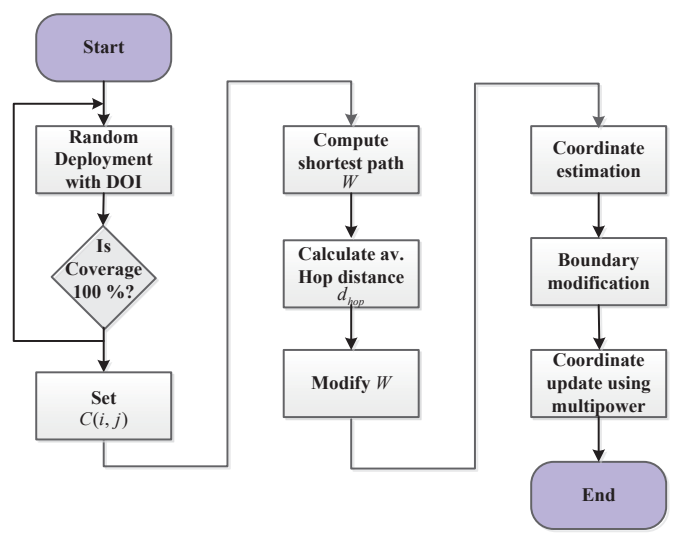

Fig. 5. (Color online) Flowchart of the proposed BIA-MMS localization method. 
(4) $\mathrm{DOI}=0.01$;

(5) Number of anchor nodes $=20$, random deployment;

(6) Grid-scan area $=2 \times 2 \mathrm{~m}^{2}$.

Figure 6 shows the average error of estimation over 35 rounds of simulations. In each round, the average error of estimation was calculated using the results of 100 deployments. The proposed BIA-MMS localization method outperformed all other methods with respect to the average error of estimation. Table 2 presents $\bar{e}_{\text {est }}$, the mean of the 35-round average errors of estimation, when various localization methods were used. The mean found when the proposed method was used was $\bar{e}_{e s t}=0.1239(\mathrm{R})$, which was the lowest among the compared methods. Table 3 presents the statistics for the error of estimation: median, interquartile range (IQR), number of outliers, and maximal outlier. Errors of estimation were divided into two categories: outliers and nonoutliers, defined by whether the error was outside the range (median $-1.5 \times \mathrm{IQR}$, median $+1.5 \times \mathrm{IQR}$ ). The furthest outliers are considered the maximal outliers. The statistics for the proposed method are superior to those for the other methods, with the exception that the number of outliers is slightly more than that for the DV-hop, grid-scan, and amorphous methods. The BIA-MMS median is 0.119 (R), which is $73.1 \%$ lower than that for the compared methods. The corresponding box-and-whisker plot of average error of estimation for each method listed in Table 3 is shown in Fig. 7.

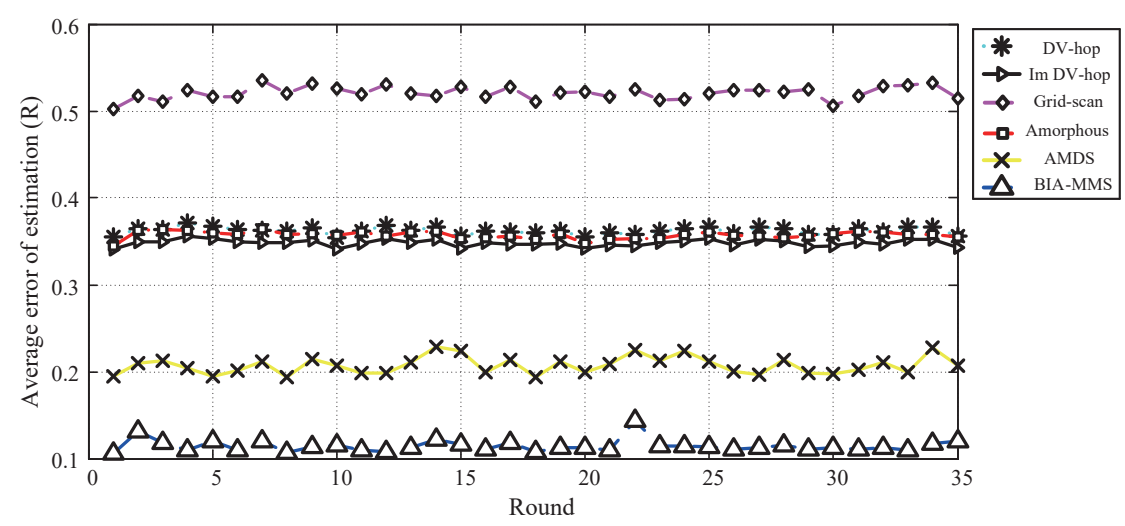

Fig. 6. (Color online) Average error of estimation over 35 rounds using various methods.

Table 2

Average error of estimation (unit: R).

\begin{tabular}{lcccccc}
\hline Method & DV-hop & Im DV-hop & Grid-Scan & Amorphous & AMDS & BIA-MMS \\
\hline $\bar{e}_{\text {est }}$ & 0.3602 & 0.3516 & 0.5057 & 0.3516 & 0.2098 & 0.1239 \\
\hline
\end{tabular}

Table 3

Statistics for the error of estimation (unit: R).

\begin{tabular}{lccccc}
\hline Item & DV-hop & Grid-Scan & Amorphous & AMDS & BIA-MMS \\
\hline Median & 0.359 & 0.507 & 0.351 & 0.206 & 0.119 \\
IQR & 0.011 & 0.21 & 0.013 & 0.012 & 0.015 \\
No. of outliers & 1 & 0 & 0 & 36 & 2 \\
Max outlier & 0.384 & 0.542 & 0.372 & 0.335 & 0.19 \\
\hline
\end{tabular}


To investigate the effect of node density on the error of estimation, the number of sensor nodes was varied from 100 to 200 . Figure 8 illustrates the average errors of estimation calculated over 100 deployments when the number of sensor nodes was changed. The proposed method again outperformed all the other methods. Table 4 lists $\bar{e}_{e s t}$, the mean of the average errors of estimation, for various localization methods, and shows that the mean for our method was $\bar{e}_{e s t}=0.1239$ (R), which was the lowest among the compared methods. When more nodes were used, the BIA-MMS method was more accurate, and the IQR remained as $0.062(\mathrm{R})$, which implies a highly uniform average error when the number of nodes was changed.

How error depended on the number of anchor nodes was investigated next. Figure 9 depicts the average error of estimation calculated over 100 deployments when the number of anchor nodes was varied from 3 to 20 . The proposed method yielded smaller average errors than other methods

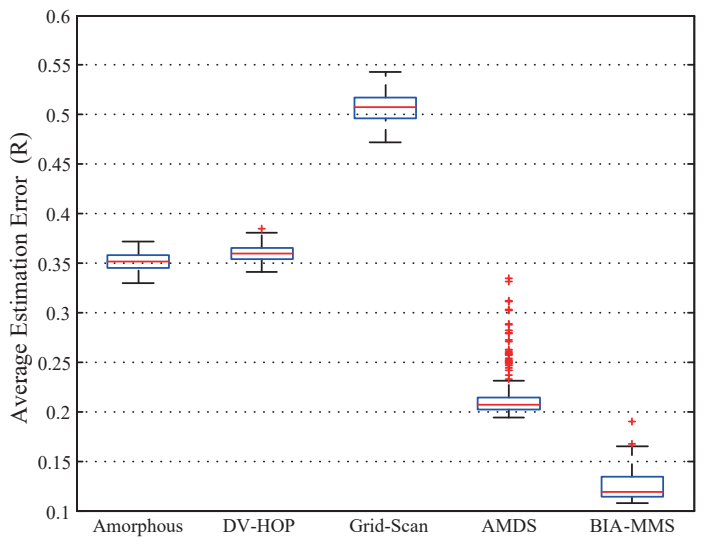

Fig. 7. (Color online) Box-and-whisker plot for different localization methods.

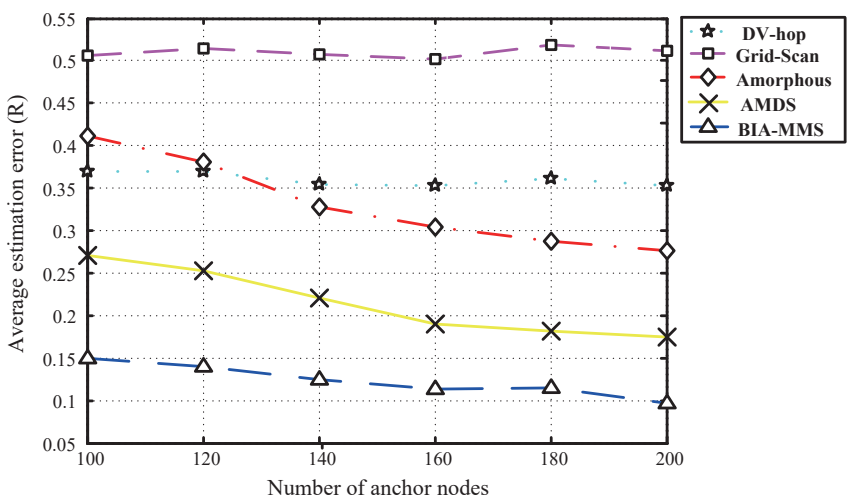

Fig. 8. (Color online) Average error of estimation when different numbers of sensor nodes were used.

Table 4

Average error of estimation over different numbers of sensor nodes (unit: R).

\begin{tabular}{lcccccc}
\hline Algorithm & DV-hop & Grid-Scan & Amorphous & AMDS & BIA-MMS & Im DV-hop \\
\hline $\bar{e}_{\text {est }}$ & 0.3598 & 0.5101 & 0.331 & 0.2098 & 0.1231 & 0.351 \\
\hline
\end{tabular}

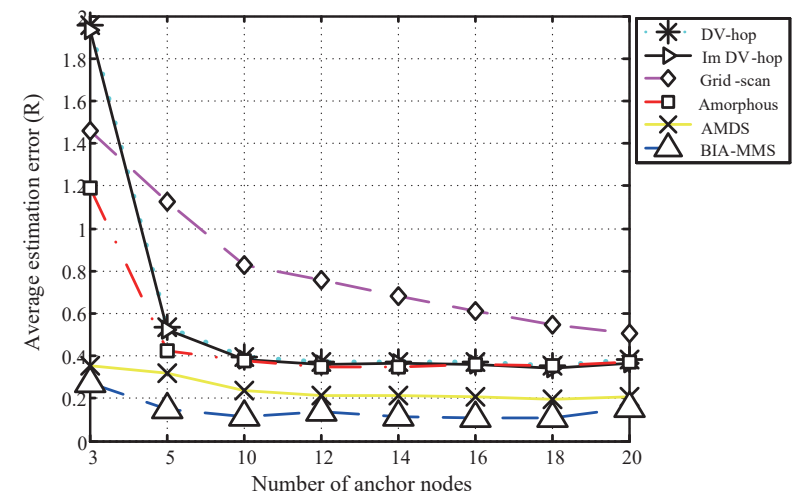

Fig. 9. (Color online) Average error of estimation when different numbers of anchor nodes were used. 
Table 5

Average error of estimation when different numbers of anchor nodes were used (unit: R).

\begin{tabular}{lcccccc}
\hline Algorithm & DV-hop & Grid-Scan & Amorphous & AMDS & BIA-MMS & Im DV-hop \\
\hline $\bar{e}_{\text {est }}$ & 0.5716 & 0.8219 & 0.4723 & 0.2530 & 0.1463 & 0.553 \\
\hline
\end{tabular}

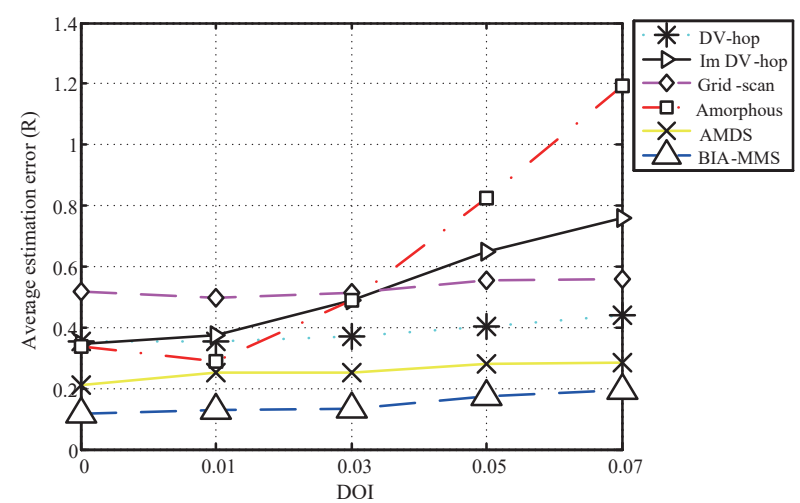

Fig. 10. (Color online) Average error of estimation for different DOIs.

Table 6

Average error of estimation for different DOIs.

\begin{tabular}{lcccccc}
\hline Algorithm & DV-hop & Grid-Scan & Amorphous & AMDS & BIA-MMS & Im DV-hop \\
\hline $\bar{e}_{\text {est }}$ & 0.3772 & 0.4807 & 0.6229 & 0.2645 & 0.1493 & 0.5236 \\
\hline
\end{tabular}

for all anchor node numbers, and the mean error when our method was used was $\bar{e}_{e s t}=0.1463$, the lowest among the compared methods as listed in Table 5.

Finally, the DOI was varied from 0.00 to 0.07 , and the numbers of sensor nodes and anchor nodes were fixed at 130 and 20, respectively. Radio irregularity affected localization accuracy substantially (Fig. 10), because irregular transmissions affect the determination of internode hop count, and the situation occurring in the detoured path leads to less accurate estimated coordinate. However, for each DOI, the proposed method still has smaller average errors than those of the other methods, as reflected in the mean errors listed in Table 6.

\section{Conclusion}

In this paper, we proposed the BIA-MMS method for estimating the coordinates of nodes in a uniformly, randomly deployed WSN using the DOI model. Assuming the network has $100 \%$ coverage, the method adopts the Floyd-Warshall algorithm to calculate the shortest path between nodes and thus the optimal hop count to avoid detour situations. Hop distance is calculated more accurately by assuming a Poisson distribution of nodes in a neighborhood. The amorphous method is also adopted to modify the average hop count. After the hop count and hop distance are found, MDS is used to calculate node coordinates. Additionally, a boundary improvement rule is used to reduce the error of estimation that occur at the boundary, and the multipower technique is introduced to further update the coordinates of nodes far from anchor nodes. Considering long-run simulations, different numbers of sensor nodes, different numbers of anchor nodes, and different DOIs, the 
proposed method obtains the best results in each case as compared with other methods. On the basis of numerical simulations, the use of the BIA-MMS method substantially reduces the average and spread of error in the estimated location in the presence of radio irregularities compared with other methods.

\section{Acknowledgments}

This research was supported by the Ministry of Science and Technology, R.O.C. (under Grant No. MOST 105-2221-E-023-063) and Long-Lite Ind. Co. Ltd. (under Grant Nos. 203A222 and 204A152).

\section{References}

1 I. F. Akyildiz, W. Su, Y. Sankarasubramaniam, and E. Cayirci: Comput. Netw. 38 (2002) 393.

2 J. M. Kahn, R. H. Katz, and K. S. J. Pister: Proc. Int. Conf. Mobile Computing and Networking (1999) 271.

3 S. C. Mukhopadhyay and J. A. Jiang: Wireless Sensor Networks and Ecological Monitoring (Springer-Verlag, Berlin Heidelberg, 2013).

4 C. Sukwon, K. Nakyoung, C. Hojung, and H. Rhan: Sensors 9 (2009) 7970.

5 H. Guesmi, S. B. Salem, and K. Bacha: Comput. Electr. Eng. 41 (2015) 226.

6 G. Werner-Allen, K. Lorincz, M. Ruiz, O. Marcillo, J. Johnson, J. Lees, and M. Welsh: IEEE Internet Comput. 10 (2006) 18.

7 P. Magana-Espinoza, R. Aquino-Santos, N. Cardenas-Benitez, J. Aguilar-Velasco, C. Buenrostro-Segura, A. Edwards-Block, and A. Medina-Cass: Sensors 14 (2014) 7096.

8 D. Niculescu and B. Nath: Telecommun. Syst. 22 (2003) 267.

9 K. Liu, X. Yan, and F. Hu: IEEE Int. Conf. Intelligent Computing and Intelligent Systems (2009) 511.

10 R. Nagpal, H. Shrobe, and J. Bachrach: Proc. 2nd Int. Conf. Information Processing in Sensor Networks (2009) 1540 .

11 L. Zhao, X. Wen, and D. Li: Int. J. Distrib. Sens. Netw. 2015 (2015).

12 Z. Xing, C. Li, and H. Li: The 27th Chinese Control and Decision Conference (2015) 1431.

13 X. Yang, M. Liu, and D. Deng: 2015 IEEE Int. Conf. Signal Processing, Communications and Computing (2015) 1.

14 C. L. Tseng, F. Y. Liu, C. H. Lin, and C. Y. Lee: Int. Symp. Computer, Consumer and Control (IS3C) (2016) paper ID: 145.

15 G. Zhou, T. He, S. Krishnamurthy, and J. Stankovic: ACM Trans. Sens. Netw. 2 (2006) 221.

16 W. Mendenhall, D. D. Wackerly, and R. L. Scheaffer: Mathematical Statistics with Applications (PWS-Kent Publishing Company, Boston, 1989).

17 L. Kleinrock and J. Silvester: Proc. IEEE National Telecommunication Conf. (1978) 4.3.1.

18 Torgerson: Torgerson's Classical MDS Derivation, http://forrest.psych.unc.edu/teaching/p230/Torgerson.pdf (accessed May 2016).

19 Y. Shang and W. Ruml: 23rd Annu. Joint Conf. IEEE Computer and Communications Societies (2004) 2640.

20 N. F. Garfield: Floyd-Warshall Algorithm (Anim Publishing, United States, 2011).

21 H. Ahn and J. Hong: Int. Conf. Ubiquitous and Future Networks (ICUFN) (2011) 7.

22 J. P. Sheu, P. C. Chen, and C. S. Hsu: IEEE Trans. Mobile Comput. 7 (2008) 1110. 